\title{
Marot, Clément.
}

Recueil inédit offert au connétable de Montmorency en mars 1538 (manuscrit de Chantilly), édition préparée par François Rigolot.

Textes Littéraires Français, 604. Genève: Droz, 2010. 353 p. + 152 pl. fac. sim. ISBN 978-2-600-01407-6 (broché) $114 €$

Les éditions modernes de la poésie de Marot prennent deux formes. Il y a les tentatives de donner l'ensemble de l'œuvre, et l'on sait à quel point une telle entreprise, en ce qui concerne les œuvres de la Renaissance, est une gageure (voir les éditions de C.A. Mayer [vol.1 : 1958], G. Defaux [1990, 1993] et récemment Fr. Rigolot [2007, 2009]). Une autre option, choisie par V.-L. Saulnier (1958), Fr. Lestringant (1987) et récemment Fr. Roudaut (2007), consiste à publier un corpus partiel. C'est alors toujours L'Adolescence, c'està-dire le premier recueil que Marot a fait imprimer de ses poèmes, en 1532, qu'on publie. En effet, L'Adolescence se justifie comme ensemble lyrique par le fait qu'elle a une économie délibérée, établie par Marot lui-même, et, fait moins anodin qu'il n'y paraît, qu'elle porte un titre en propre. Par contraste, la plupart des publications qui ont suivi, à l'instar de La Suite (1534), se présentent le titre de ce deuxième recueil le met bien en évidence - comme un ajout à L'Adolescence, un " allongeail » pour parler comme Montaigne, si bien qu'il est difficile de publier un recueil de poèmes de Marot qui ne soit L'Adolescence elle-même ou une version augmentée de celle-ci, à l'exception peut-être des Epigrammes ou des traductions (Métamorphoses d'Ovide, Psaumes) qui ont une logique d'ensemble, mais qui ont l'inconvénient de ne pas rendre entièrement justice au génie et à l'esthétique de Marot, qui tiennent en grande partie à sa versatilité. Pour les raisons qu'on vient d'exposer, l'édition que François Rigolot donne aujourd'hui du manuscrit de Chantilly apporte un souffle nouveau aux études marotiques. Elle était d'ailleurs attendue ; Gérard Defaux, l'avait appelée de ses vœux lorsqu'il travaillait sur sa propre édition des œuvres complètes de Marot.

Ce manuscrit d'apparat, composé de cent quarante pièces et assemblé en 1538, a l'avantage, comme L'Adolescence, d'être un ensemble cohérent, composé par Marot lui-même et qui met en évidence toute la palette des talents de l'auteur. Rappelons le contexte qui entoure ce manuscrit. Suite à l'affaire des placards (1534), qui avait déclenché une répression contre ceux qui contestaient l'orthodoxie religieuse, Marot s'enfuit dans le nord de l'Italie, à Ferrare, 
où il reçut le soutien de Renée de France. Il y poursuivit ses activités poétiques, lança le fameux concours des blasons, se plongea dans les Tristes d'Ovide et adressa des lettres au roi et au dauphin pour obtenir leur clémence. En 1536, après un détour par Venise, le poète retourna en France, fit amende honorable à Lyon et retrouva sa place à la cour de François I ${ }^{\text {er }}$. En 1538, il offre un manuscrit de ses œuvres récentes, c'est-à-dire composées pendant son exil et après celui-ci, au connétable Anne de Montmorency, qui est alors, comme on dit, l'homme fort du régime. Le manuscrit est intitulé Recueil des dernières ouvres de Clément Marot non imprimées et premièrement celles qu'il fit durant son exil et depuis son retour. "Rien de plus intéressant que ce manuscrit », disait C.A. Mayer ${ }^{1}$. Certaines pièces bien connues de l'auteur figurent dans ce recueil, telles que "L'épître de Venise », "Les Adieux de Marot à la ville de Lyon » ou " Le blason du beau tétin ». L'ensemble est extrêmement cohérent puisqu'il s'organise autour de l'exil de Marot et en est en même temps, en quelque sorte, la chronique, la dispositio répondant globalement à un principe chronologique et subsidiairement générique. Pour tous ceux qui fréquentent Marot, la valeur de ce recueil saute aux yeux : il apporte une contrepartie bienvenue à L'Adolescence et constitue un ensemble tout aussi cohérent, mais cette fois pour l'œuvre de la maturité (1535-1538). Les poèmes ainsi réunis sont tout à fait représentatifs de l'infinie créativité de Marot et de ses préoccupations du moment, puisqu'on y trouve entre autres pièces de circonstance, littérature de voyage, blasons, épigrammes, élégies, gauloiseries, etc. Il faut ajouter que la plupart des pièces reproduites dans le recueil étaient jusque-là inédites - certaines le resteront d'ailleurs jusqu'au $\mathrm{XX}^{\mathrm{e}}$ siècle - , ce qui souligne la valeur du don fait au connétable et l'importance du manuscrit pour la critique.

Le seul inconvénient de ce recueil, signalé dès les premières études modernes sur le corpus marotique ${ }^{2}$, tient à la personnalité de son destinataire : le connétable de Montmorency était connu pour son conservatisme religieux, ce qui a incité Marot, qui voulait éviter de le heurter, à exclure certaines de ses compositions d'exil les plus polémiques; c'est ainsi que la fameuse « L'Épître au roi, de Ferrare », dans laquelle le Quercinois se justifie d'avoir eu en sa possession des textes condamnés, de même que les « coq-à-l'âne » écrits à cette 
époque ne figurent pas dans le recueil. Pour la même raison, Marot a adapté, adouci certains des poèmes reproduits - heureusement l'ampleur des ces adoucissements s'avère, après lecture, moins grande qu' on pouvait le craindre -, ce qui explique qu'ils offrent des versions qui ne sont pas celles retenues par les éditions ultérieures ; "Lépître de Venise ", par exemple, se trouve dans la version du manuscrit expurgée de ses passages aux accents les plus luthériens.

On peut bien entendu déplorer ce travail d'autocensure ; mais on peut aussi s'interroger, avec François Rigolot, sur le réflexe critique qui nous pousse en général à préférer les versions polémiques à celles qui le sont moins : certes, personne ne doute des convictions réformistes de Marot, mais la question demeure : quand il lance des diatribes extrêmement violentes, exprime-t-il purement et simplement son sentiment profond ou force-t-il un peu le trait pour plaire à ses protecteurs réformistes? Difficile pour nous de décider qui était le "vrai Marot », si tant est qu'une telle vérité existe.

En raison de ce travail d'autocensure, le recueil de Chantilly n'est pas l'œuvre d'exil au complet qu'on souhaiterait - quel Marotiste ne s'émerveillerait d'un tel recueil qui inclurait les pièces de celui-ci, non expurgées, augmentées des «coq-à-l'âne » et de "L'épître au roi, de Ferrare » ? —, mais il a l'avantage d'exister, et de constituer un échantillonnage très riche de la production de Marot entre 1534 et 1538, ainsi qu'un objet littéraire très stimulant pour les études marotiques.

L'édition donnée ici par François Rigolot est un objet magnifique. Outre le texte en transcription, l'ouvrage comporte un fac-similé de l'ensemble du manuscrit, ainsi qu'une mise en contexte et un commentaire très éclairant des différentes sections du recueil. L'ouvrage se clôt sur une annexe très utile ; on y trouve la version "standard " des poèmes qui, dans le manuscrit, ont été expurgés ainsi que d'autres pièces qui se rattachent au recueil, point de départ de certaines compositions ou réponses à celles-ci.

Cette initiative novatrice illustre l'intérêt qui est celui de l'éditeur et de nombreux critiques de nos jours pour l'étude des textes considérés dans leur habillage originel, c'est-à-dire à travers les éditions de l'époque, qui ont pour caractéristique d'être toujours partielles, mouvantes et ouvertes à l'hétérogénéité. Au rebours des éditions qui visent à établir un texte de référence, l'intention de Rigolot est simplement ici, comme il le déclare lui-même, de mettre en lumière un moment de la carrière de Marot et de l'histoire des lettres françaises ; geste original qui a en outre le poids de la rigueur scientifique. Dans le cadre 
des études marotiques on peut rapprocher cette parution de l'édition donnée par Defaux des Fleurs de Poesie Françoyse en $2002^{3}$; cette dernière illustre en effet la même approche dans la mesure où elle reproduit un type d'ouvrage que les éditeurs modernes choisissent en général d'ignorer, c'est-à-dire les ouvrages collectifs, qui bousculent notre perspective auctorio-centrique, mais qui étaient très courants et avaient une existence propre à la Renaissance. On ne peut que souhaiter que de telles publications en appellent d'autres; on pense, par exemple, au recueil illustré des Blasons du Corps Femenin de 1536 ou au magnifique Champfleury de Geoffroy Tory, qui eux aussi mériteraient une édition (ou une réédition).

FLORIAN PREISIG, Eastern Washington University

\section{Molins, Marline.}

\section{Charles Fontaine Traducteur. Le Poète et ses mécènes à la Renaissance.}

Travaux d'Humanisme et Renaissance, 491. Genève: Droz, 2011. 367 p. ISBN 978-2-600-01509-7 (broché) \$108.

Le présent ouvrage publié par M. Molins constitue la version remaniée de sa thèse de doctorat (Traduction et narration à la Renaissance) soutenue à l'Université de Lille en 2003. Il se concentre ici sur l'œuvre de traducteur du seul Charles Fontaine, poète parisien et auteur d'une production poétique abondante et trop souvent méconnue. C'est l'un des mérites de cette étude critique que de sortir de l'ombre un écrivain prolifique dont il fallait réévaluer la place dans le paysage littéraire français. En effet, la seule monographie qui lui a été consacrée date de près d'un siècle (R. L. Hawkins, Maistre Charles Fontaine parisien [Cambridge, Harvard University Press, 1916]), et malgré les quelques pages qui lui accorde M. Raymond (L'Influence de Ronsard sur la poésie française [Genève : Droz, 1965], p. 56-63) ou encore les rares études qui furent publiées depuis (citons celle de R. Scalamandrè, en 1970, et les actes à paraître du colloque qui s'est tenu à Lyon en juin 2009), beaucoup reste à explorer dans ce parcours d'un des 